\title{
Early Career Members at the ERS LSC 2017: mechanistic overlap between chronic lung injury and
} cancer

\section{Early Career Forum}

The 15th ERS Lung Science Conference (LSC) took place in Estoril, Portugal, on March 23-26, 2017. More than 180 delegates from across the globe gathered to present their work, and have the opportunity to discuss and interact with peers and mentors. The focus for this year was "mechanistic overlap of chronic lung injury and cancer".

Many young researchers from all over the world attended to learn from experts in the field as they presented on topics including:

- pathological and mechanistic disease similarities

- intersecting immunity and inflammation

- modelling disease with organoids

In this article, we summarise some scientific highlights from this year's conference.

\section{Disease similarities, shared repair pathways and stromal synergies}

The LSC was kicked off by the opening lecture given by Gary Anderson from The University of Melbourne (Melbourne, Australia), who elegantly introduced state-of-the-art research on lung cancer development. He specifically highlighted that patients suffering from chronic obstructive pulmonary disease (COPD) have a massively increased risk of developing lung cancer over time. This clearly indicates the important contribution of inflammation to cancer development, a point that would be addressed by many others in the lectures that followed.

Basal cells are very important contributors to lung cancer and represent the bronchial airway stem cell population in the lung. While in healthy lungs, these cells are involved in regeneration of injured tissue, they can also give rise to precancerous lesions. This is especially important, as Sam Janes (University College London, London, UK) showed that environmental chemical noxes such as cigarette smoke can induce genetic alterations in basal cells that persist long after smoking cessation. Thus, understanding the detailed regulatory mechanisms would hold great potential for therapy of various lung diseases. Along this line, in the session entitled "Shared Pathways and Repair", Carla Kim from the Boston Children's Hospital and Harvard Medical School (Boston, MA, USA) reported data from single-cell sequencing studies that identified distinct populations of airway stem cells as either being Lgr5 ${ }^{+}$or Lgr6 ${ }^{+}$. Interestingly, a genetic depletion of Lgr6+ cells impaired the regenerative capacity of those cells.
Cite as: Almendros I, Bartel S, Bostantzoglou C, et al. Early Career Members at the ERS Lung Science Conference 2017: mechanistic overlap between chronic lung injury and cancer. Breathe 2017; 13: 323-326. 
Another important player in lung diseases such as COPD, lung fibrosis and lung cancer are stromal cells, which were discussed in the first session on March 25, 2017: "Stromal Similarities and Synergies". Saverio Bellusci from The University of Giessen (Giessen, Germany) highlighted the importance of different types of fibroblasts (the lipogenic and myogenic fibroblast phenotypes) in the lung and their potential contribution to lung fibrosis, while Angela Nieto (Neurosciences Institute, Alicante, Spain) focused on the conundrum of epithelial-to-mesenchymal transition in development and disease.

\section{Intersecting immunity and inflammation}

The session "Intersecting Immunity and Inflammation" started with Marie-Caroline Dieu-Nosjean from the Centre de Recherche des Cordeliers de Jussieu in Paris (France), who showed the potential role of tertiary lymphoid structures (TLSs) in cancer progression. The presence of TLSs in non-small cell lung cancer has been associated with a better prognosis in this type of lung cancer. In particular, TLSs seem to be crucial for tumour surveillance by facilitating lymphocyte recruitment to the tumour stroma and enhancing the T- and B-cell responses as well as Th1 polarisation. Therefore, further studies focused on TLSs, aiming to increase their production, could represent a novel therapeutic strategy to reduce the metastatic capacity of several types of cancer.

In the second presentation, Christoph Beisswenger (Saarland University, Homburg, Germany) showed that lung infection could increase lung adenocarcinoma proliferation through Toll-like receptor (TLR) regulation. Specifically, they exposed wild-type (WT) and TLR-knockout mice to non-typeable Haemophilus influenzae in order to induce lung infection. Interestingly, they found that only WT mice experienced increased tumour proliferation resulting in bigger tumours respect to TLR-knockout mice. Thus, these results could be relevant to COPD which has been well characterised by chronic inflammation and recurrent lung infections.

Magda Spella (University of Patras, Patras, Greece) has shown how myeloid activation of the nuclear factor (NF)- $\kappa B$ pathway can increase the chance of KRAS mutations. Therefore, her results suggest that the pro-inflammatory NF- $\kappa B$ pathway, by promoting increased numbers of $K R A S$ mutations, could explain the higher malignancy and resistance to conventional anticancer drugs encountered in some types of tumours.

In the last presentation, Karim Vermaelen (University of Ghent, Ghent, Belgium) exposed the importance of immunomodulation of dendritic cells (DCs) and other immune cells in lung cancer. By employing a mouse model of lung cancer, he showed how the lung tumour micro-environment can alters the tissue-resident DC populations.

\section{Posters}

This year's LSC featured 76 poster presentations divided into two sessions. While all poster presentations were scientifically excellent, here, we highlight a few. This session included a presentation by Alexandra Schröder (Research Center of Borstel, Borstel, Germany) on the role of interleukin (IL)-37 during experimental asthma. Her results suggested that IL-37 ameliorates asthma through interacting with IL-18 receptor- $\alpha$ and the single-immunoglobulin IL-1-related receptor. She suggested that IL-37 utilises and forms a complex with both receptors to inhibit pro-inflammatory signalling. These results could have profound effects for asthma treatment.

A brilliant presentation came from the field of lung cancer by Michela Terlizzi (Università degli Studi di Salerno, Fisciano, Italy). Using bronchoalveolar lavage-derived tumour-associated macrophages (TAMs), she showed how these promote tumour activity. She proved that their pro-tumour activity was driven via activation of the NLRP3 inflammasome with caspase 1 activation and $\mathrm{IL}-1 \alpha$ and $\mathrm{IL}-1 \beta$ release. She highlighted how inflammasome activation was mitochondrial reactive oxygen species (mtROS) production-dependent, and how mtROS production was driven by caspases 1 and 11. Depletion of $\mathrm{IL}-1 \alpha-$ and IL-1 $\beta$-producing TAMs in vivo reduced lung tumourigenesis. Therefore, it would appear that during lung cancer progression, TAMs are activated and respond to the insult in a way that drives tumour progression. This centres around an interplay between inflammatory caspases, the mitochondria and the NLRP3 inflammasome, and studying this could offer a novel therapeutic target. This poster was awarded the Distinguished Prize.

\section{Young investigator session: the William MacNee Award}

During this year's young investigator session, which is also a competition for the William MacNee Award, research of high scientific quality was presented, covering various pulmonary diseases. Anthi Krontira (Medical School, University of Patras) presented data on a pathway through oncogenic Kras and tp53 mutations that leads to development of malignant mesothelioma. Nikolaos Kanellakis' research (University of Patras) on mouse lung adenocarcinoma cell lines, obtained by chronically exposing various mouse strains to different tobacco carcinogens, provides a model of human lung adenocarcinoma allowing characterisation for novel cancer oncogenes. Amal Houssaini (Helmholtz Zentrum München, Munich, Germany) presented data from research on the mTOR signalling pathway, which is found to be strongly activated in lungs from patients with COPD and may represent a potential target to inhibit lung cell senescence in COPD. 
The next presentation was given by Gizem Günes (Munich Medical Research School, Munich), whose work implicates the epigenetic regulator HMGN5 in COPD resulting from chronic lung injury. Finally, Xiang Zheng (Max Planck Institute for Heart and Lung Research, Bad Nauheim, Germany), who was received the William MacNee Award, presented his work on the epigenetic mechanisms of macrophage polarisation and re-education of TAMs by modulating histone deacetylases in lung cancer, providing evidence that suppression of histone deacetylases regulates tumour cell functions, which therefore may offer a novel strategy on cancer therapy.

\section{Other awards}

The ERS presented awards to eight distinguished poster presentations and the best of seven selected oral presentations. Overall, we want to congratulate all the presenting authors and their research teams for the high level of science that was presented, enabling fruitful discussions between the audience and the presenting scientists.

\section{Junior session: successful funding applications and opportunities}

This year's junior session aimed to help young investigators gain a better understanding of funding opportunities. The session included four speakers with successful track records of obtaining research grants. All speakers encouraged junior scientists to apply for research funding, and gave their own personal advice and insights into what they thought contributed to their success.

The session was opened by Rory Morty from the University of Giessen, who is currently the Fellowships and Awards Director of the ERS. He emphasised the importance of supporting junior investigators to the ERS. He provided an overview of the different ERS funding mechanisms and emphasised that the ERS fellowships have a very high success rate ( $40 \%)$ when compared to other sources of funding. He thus encouraged young investigators to take advantage of this unique opportunity and to apply for ERS fellowships. Dr Morty urged future applicants to not overstate achievements but encouraged them to highlight their leadership experiences in their applications and to actively participate in societies.

His talk was followed by Georgios Stathopoulos (Comprehensive Pneumology Center (CPC) at Helmholtz Zentrum München) who, as a European Research Council (ERC) Starting Grant awardee, talked about funding via the ERC. Dr Stathopolous began his talk by giving an overview of the ERC's mission to raise the visibility of European research and support "bottom-up" innovative research. By almost any measure, this programme has been overwhelmingly successful. There are four different grant mechanisms currently offered by the ERC: the three different major grants (Starting, Consolidator and Advanced) span nearly the entire career path of investigators, while the Proof of Concept grants are available to any ERC grant holder to help bridge the gap between research and early marketing stages. While the funding rates have averaged around 10\%, Dr Stathopoulos shared his own personal experiences and encouraged everyone to submit their applications. He emphasised that the innovation of the project is an extremely important component to the ERC grant application.

Next, Janette Burgess from the University of Groningen (Groningen, the Netherlands) talked about funding opportunities focused on flexible research careers. From her experience, she emphasised the importance of knowing what you want and making strategical choices to achieve these goals. She encouraged young trainees to make sure that they have a curriculum vitae that stands out from the pool by having, for example, small grants or awards early in your career. Having a good mentor who can help you identify those opportunities is of great importance but additionally, you have to actively search for them.

The junior session ended with a presentation from Melanie Königshoff (University of Colorado, Denver, CO, USA) about writing a successful grant application. In order to enhance your chances of success, it is important to know which funding mechanisms are available, and best fit your personal background and goals, and your career stage. The most important part of every grant is the idea behind it; it is thus important to involve experts who can help you develop your idea along the way. In addition, you should be familiar with all eligibility and guidance criteria, stick to them throughout the process, and plan plenty of time for writing. Dr Königshoff ended her presentation by stressing the fact that everybody will have grants and papers rejected, and that you have to find a personal way to deal with this disappointment and continue trying. She left us with her personal coping strategy according to Indiana Jones: "Nothing shocks me. I'm a scientist."

One of the most difficult and critical steps for postdoctoral researchers is to transition to independence. The discussion that followed the talks focused on the realities of funding and training for young investigators in transition to independence. While the young investigators felt that there are very good training and funding mechanisms in place for postdoctoral scientists early in their career, they feel there is a gap in training opportunities when it comes to transitioning to independence. This was also recognised by more senior investigators. and the idea of special training and mentoring of former and present ERC starting grant applicants was discussed as a way to support junior investigators, and to increase success rates for ERS fellows in these applications. 


\section{Join us next year}

Once again, the LSC in Estoril proved to be an outstanding place for networking, and to promote and communicate science. Therefore, we hope to see all of you next year at LSC 2018, which will focus on the extracellular matrix.

\section{Conflict of interest}

None declared. 